\title{
processus local de destruction des roches par un outil de forage
}

\author{
par \\ Minh Duc Nguyen \\ Ingénieur Civil des Ponts et Chaussées \\ Docteur ès Sciences \\ Laboratoire de Mécanique des Solides \\ (École Polytechnique Palaiseau)
}

\section{Notations}

$$
\begin{aligned}
& \text { e }=\text { Largeur de l'outil } \\
& \mathrm{t}=\text { Profondeur de coupe } \\
& \rho=\text { rayon de courbure de l'arête de coupe } \\
& \gamma \quad=\text { angle de coupe (valeur algébrique) } \\
& \beta \quad=\text { angle de dépouille (valeur algébrique } \\
& \mathrm{F}_{\mathrm{c}}, \mathrm{F}_{\mathrm{n}}=\text { efforts de coupe tangentiel et normal }
\end{aligned}
$$

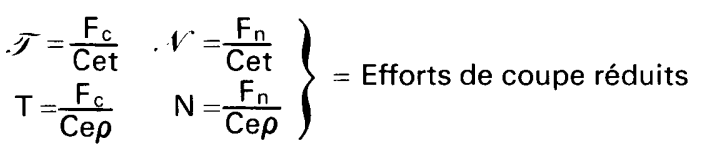

$$
\begin{aligned}
& \tau_{\mathrm{nt}}, \sigma_{\mathrm{n}}=\text { contraintes de contact } \\
& \lceil\vec{v} \mid=\text { vitesse relative du matériau au contact } \\
& \mathrm{C}=\text { cohésion ju matériau } \\
& \varphi \quad=\text { angle de frottement interne }\}_{\text {de Coulomb }} \\
& \mathrm{H} \quad=\mathrm{C} \operatorname{cotg} \varphi \text {. }
\end{aligned}
$$

\section{Introduction}

Sur un outil de forage rotatif où la destruction de la roche est obtenue par un effet de coupe, ou d'usure, ou de rayure, l'élément actif (lame, prisme, diamant...) présente des formes diverses provenant par exemple du fait de la dureté de la roche qui ne permet pas d'atteindre une profondeur de coupe suffisante pour qu'une arête de coupe puisse être considérée comme vive. De même, l'usure de l'outil entraîne des modifications de forme, et la notion d'outil en forme de dièdre (fig. 1-a) n'est qu'une première approximation.

Une étude expérimentale détaillée du processus de destruction du matériau par de tels objets est possible par une méthode de similitude utilisant des matériaux équivalents tendres, ce qui permet de s'affranchir de certaines difficultés rencontrées dans les essais en vraie grandeur $[6,7]$. On rappellera les conditions de la similitude basées sur:

- L'homothétie par rapport à l'origine de la courbe intrinsèque de rupture, approchée, dans la zone intéressée

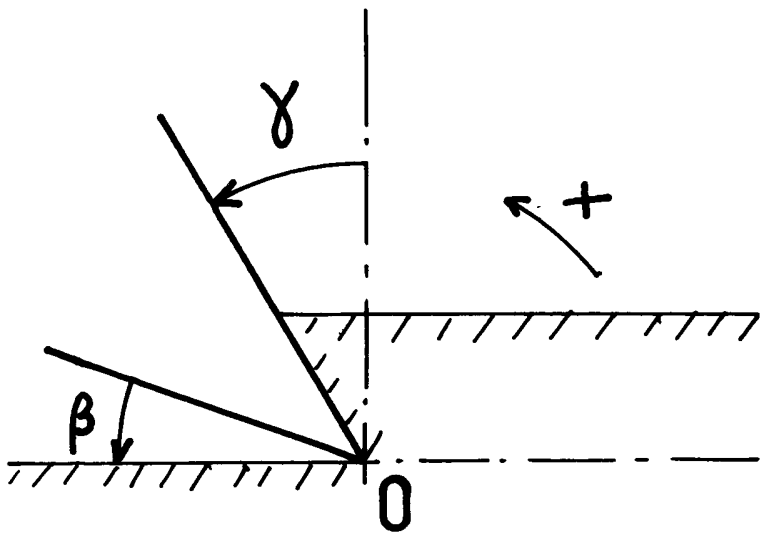

$1-a$

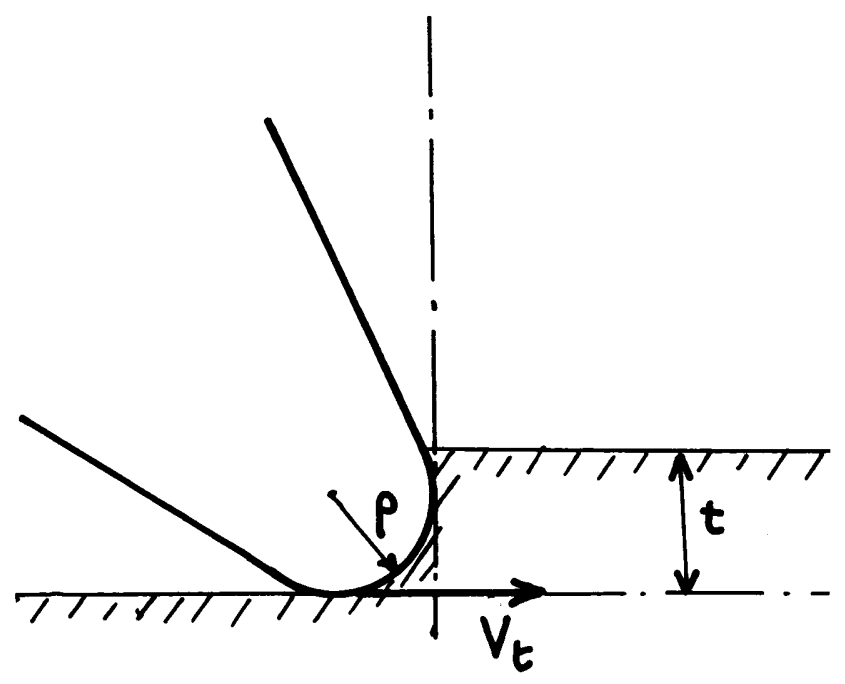

$1-b$

Fig. 1 Modèles d'outils 
par les contraintes, par un critère de Coulomb (Cohésion C, angle de frottement interne $\varphi$ ). Le théorème des états correspondants permet de transposer les résultats sur maquettes, à la pression atmosphérique, à ceux de la roche naturelle en condition de fond (c'est-à-dire sous la pression hydrostatique due à la boue), en considérant que cette roche est soumise à une pression effective de confinement $\rho_{\theta}$.

- Le respect de la ductilité globale du matériau à la pression $\mathrm{P}_{e_{1}}$ définie par le coefficient de plasticité $\mathrm{C}_{\mathrm{pl}}$ de Schreiner [9].

On présente ici l'étude d'un outil élémentaire rigide, en coupe orthogonale, avec une arête de coupe arrondie, de rayon $\rho$ (fig. $1-b$ ); son angle de coupe $\gamma$ est nul, et sa dépouille $\beta$ positive. Le choix de ce modèle ne limite en rien la généralité du phénomène qualitatif observé.

On sera amené à comparer cet outil, défini par le paramètre géométrique $\rho / t(t=$ profondeur de coupe), avec l'outil "angulaire» (fig. 1-a) défini par son angle de coupe $\gamma$, en valeur algébrique (arête 0 vive).

\section{2 Étude expérimentale}

Le matériau utilisé est un mélange de sable et de paraffine, dont quelques caractéristiques sont données dans le tableau 1.

Ce matériau, relativement ductile $\left(C_{p}=3\right)$, est susceptible de représenter une roche dans les conditions du forage pétrolier profond. L'outil se déplace dans un plan vertical (fig. 1) avec une vitesse $\vec{V}_{t}$ horizontale constante. La figure 2 représente le schéma expérimental.

\subsection{Aspects de la destruction}

Les aspects de la destruction, observés sur les outils " angulaires ", se retrouvent ici de la façon suivante :

\subsubsection{La ductilité apparente augmente avec $\rho / t$}

En effet, lorsque t est petit (fig. 7-a et début de courbe sur la fig. 7-b), les variations relatives de l'effort en fonction du temps sont faibles, et correspondent à la formation de petits débris. Au contraire, lorsque l'enfoncement t est suffisant (fig. 7-b) on reconnaît l'allure caractéristique de cet effort en « dents de scie ", liée à la formation de grands éclats; ce phénomène était reconnu pour des matériaux réputés fragiles $[3,4,8 \ldots]$.

2.1.2 L'écoulement relatif du matériau en contact, qui se fait dans des sens opposés, de part et d'autre d'une zone d'agglomération du matériau (fig. 3 et 4). A titre de comparaison ont été représentés, également (fig. 3 ), les schémas d'écoulement constatés sur les outils "angulaires " [6]. L'écoulement vers l'arrière crée une couche de matériau broyé compacté au fond du sillon, constatée également par Garner [1] sur des roches sous confinement rayées par un diamant; ce dernier attribuait ce phénomène à une " plästification » de la roche.

\section{Fig. 4 Agglomération de matériau sous l'outil}

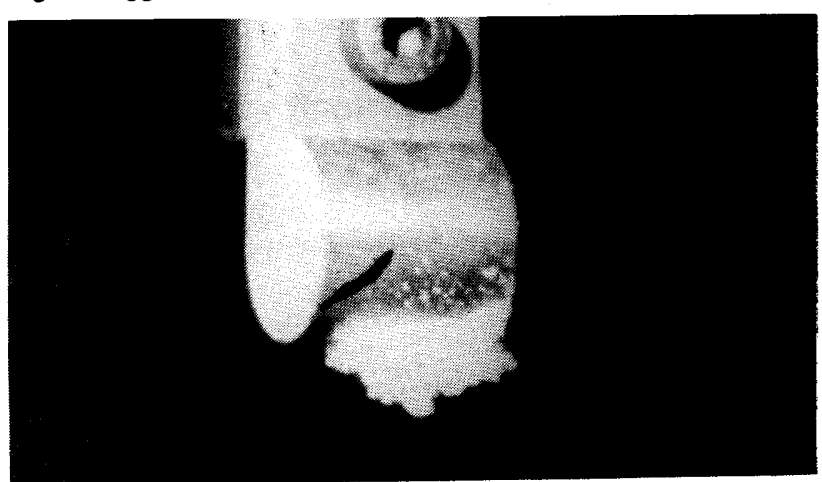

Caractéristiques du matériau :

Sable fin de Fontainebleau + Paraffine (10\% en poids de sable)

$\begin{array}{lr}\text { Masse volumique } & 1,75 \\ \text { Porosité } & 7 \% \\ \text { Coefficient de plasticité Cpl }{ }^{*} \text { ) de Schreiner } & 3 \\ \text { Module d'Young (MPa) } & 260 \\ \text { Résistance } & 1,5 \\ \text { (MPa) } & \text { Compression simple } \\ \text { Traction (essai Brésilien) } & 0,2 \\ \text { Poinçonnement } & 11,5 \\ \text { Courbe intrinsèque \{ Angle de frottement interne } & 0,5 \\ & 22^{\circ} \\ \text { (*) } \mathrm{Cpl}=\frac{\text { Énergie de destruction }}{\text { Energie élastique correspondante }} & \end{array}$

Tableau 1

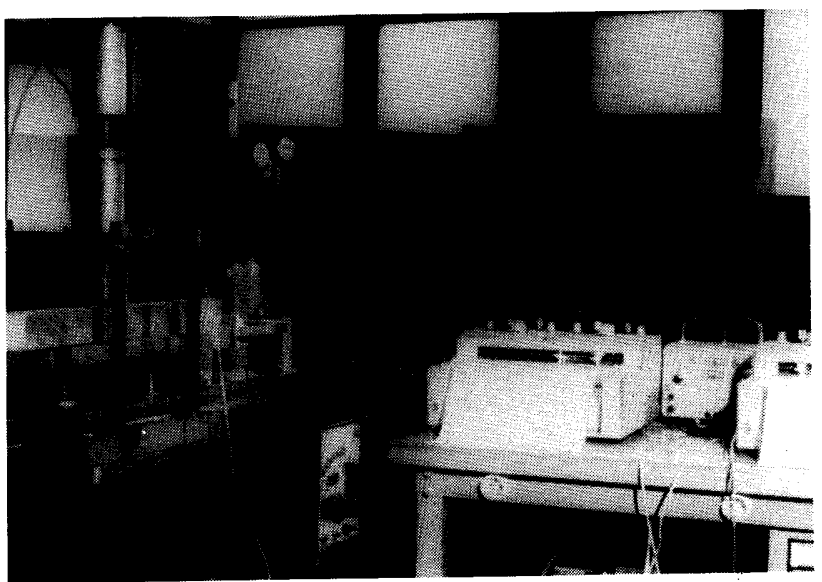

Fig. 2 Montage expérimental

a.

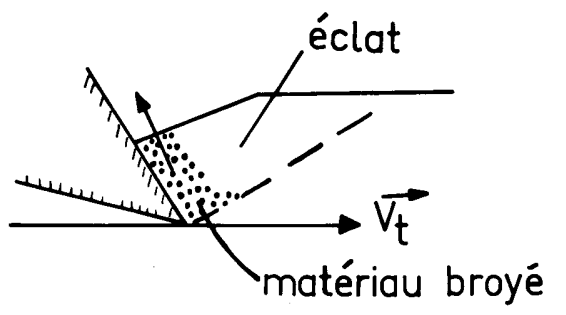

b.



Fig. 3 Schémas composés d'écoulement sous l'outil 


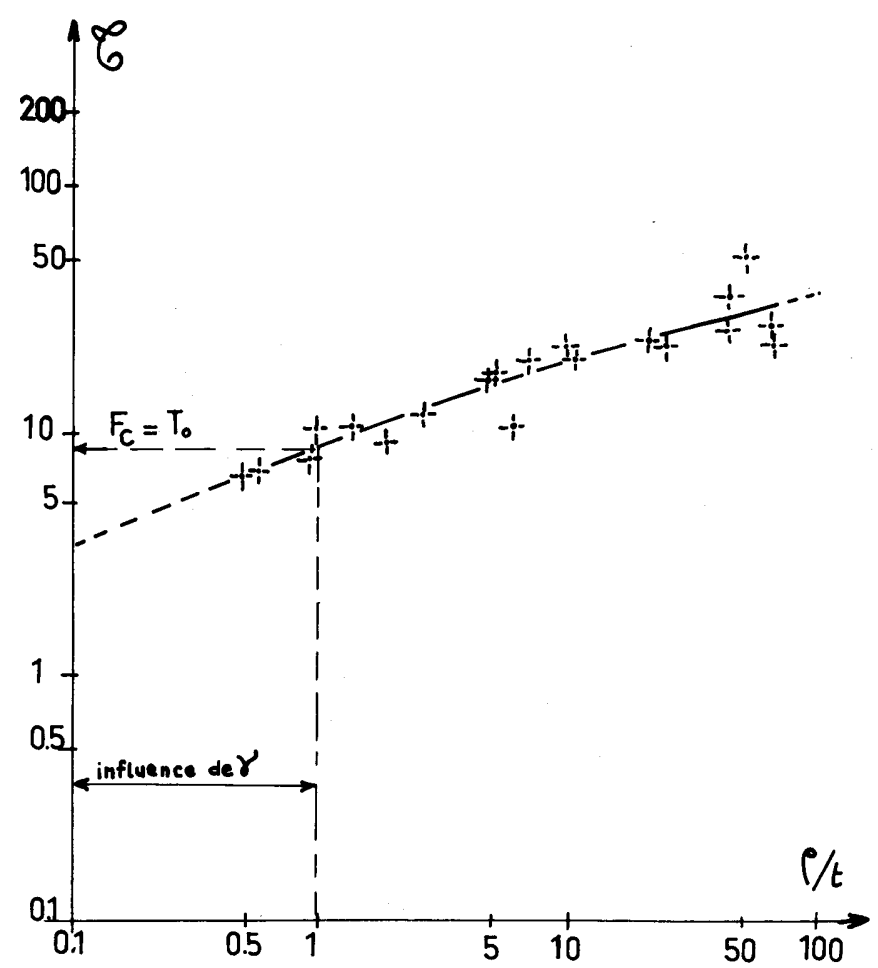

Fig. 5 Variation de. $\bar{J}$ avec $\rho / t$

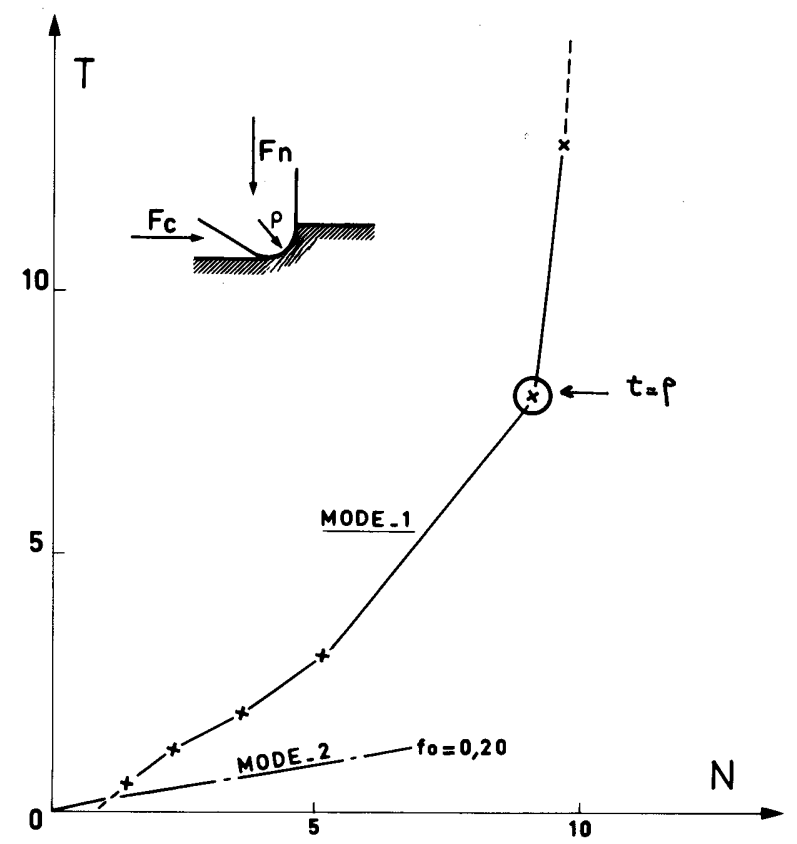

Fig. 7 Variation de $T$ et $N$

\subsection{Efforts de coupe}

Pour faciliter l'interprétation des résultats on s'intéresse ici aux valeurs maximales de coupe, en déformation plane. Ceci est obtenu pratiquement en observant un rapport profondeur de coupe sur largeur d'outil inférieur a 0,3. La précision des résultats a nécessité l'utilisation d'outils de rayons $\rho$ différents $(2 ; 1 ; 0,3 \mathrm{~cm} .$.$) .$

Les efforts sont présentés sous une première forme réduite (fig. $5 \& 6$ ) :

$$
\mathscr{T}=\frac{\mathrm{F}_{\mathrm{c}}}{\mathrm{Cet}} \quad \mathscr{N}=\frac{\mathrm{F}_{\mathrm{n}}}{\mathrm{Cet}}
$$

qui indique le rendement de la destruction en fonction de $\rho / t$ : on remarquera, en effet, que la valeur moyenne de $\mathscr{T}$ représente l'énergie fournie sur le volume détruit (pour une cohésion C unité), parfois appelée "énergie spécifique» [2].

Comme il est indiqué sur la figure, l'angle de coupe $\gamma$ n'intervient évidemment pas tant que la profondeur du

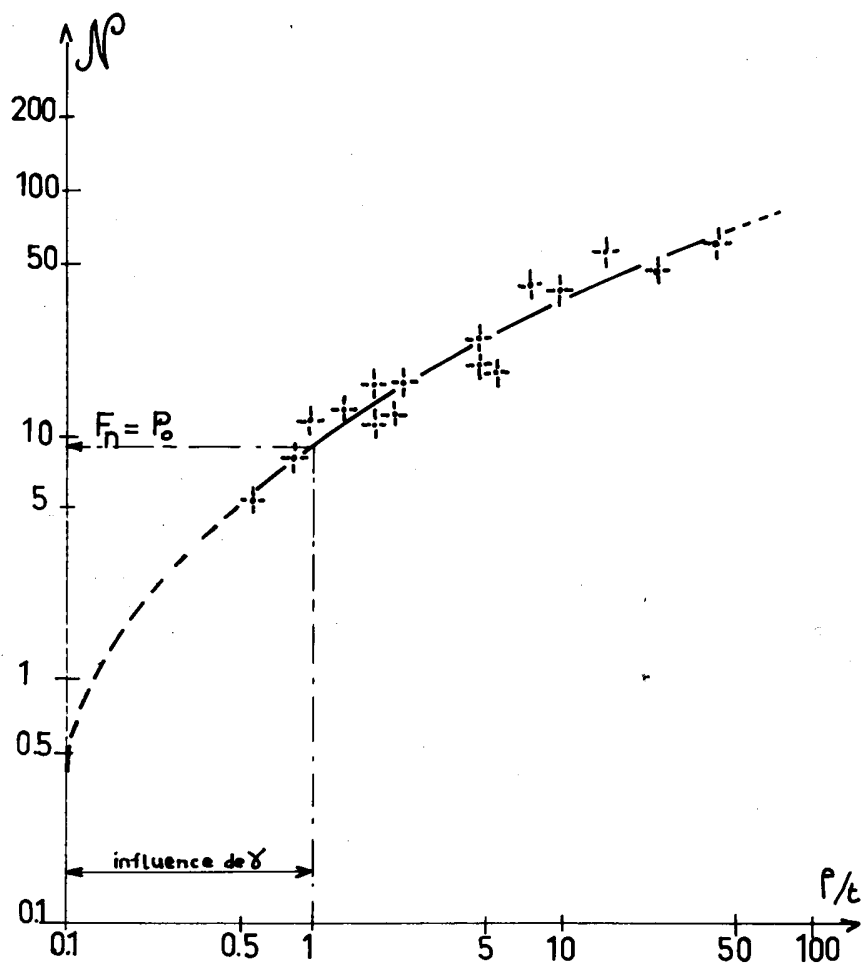

Fig. 6 Variation de $\mathfrak{N}$ avec $\rho / t$

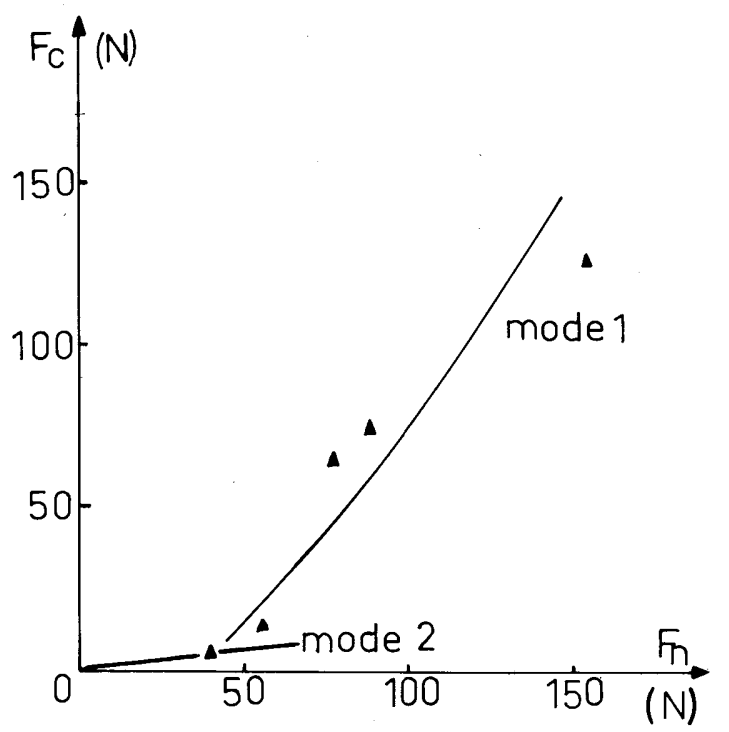

Fig. 8 Essai de rayure du verre par un diamant [5]

contact (pratiquement égale à $t$ pour notre matériau) n'excède pas la zone de courbure de l'arête, soit $t \leqslant \rho$ [ou $t \leqslant \rho(1+\sin \gamma)$ si $\gamma=0]$.

L'augmentation rapide de $\mathscr{T}$ et $\mathcal{A}$ àvec $\rho / \mathrm{t}$ doit atteindre une valeur maximale finie : en effet, lorsque $\rho / t$ est suffisamment grand, l'outil est assimilable à un plan faiblement incliné sur sa direction d'avancement et travaille alors suivant un mode différent, "abrasion " ou " mode 2 ", ainsi désigné par opposition au cas général (" mode 1 » ou " coupe ") $[6,7]$.

Pour un matériau donné, les valeurs correspondantes de.T et. $\mathscr{V}$ dépendent du frottement $f$ de contact ; elles valent ici $(f=0,20)$ :

$\widetilde{T}_{\text {MAX }}=128 \mathscr{N}_{\text {MAX }}=640$

La différenciation des deux modes de rupture précédents se retrouve sur la courbe $F_{c}$ fonction de $F_{n}$ (fig. 7).

On constatera l'allure tout à fait similaire d'une courbe analogue obtenue dans un essai de rayure d'une plaque de verre par un diamant sphérique (fig. 8 , tirée de [5]). 
La présentation des efforts de coupe sous une deuxième forme réduite :

$$
T=\frac{F_{c}}{\operatorname{Ce} \rho} \quad N=\frac{F_{n}}{\operatorname{Ce} \rho}
$$

indique la variation de ces efforts avec l'enfoncement $t / \rho$ pour un outil donné (fig. 9).

L'augmentation de ces efforts avec $t$ est rapide jusqu'à $t=\rho$, et est ensuite comparable à celle d'un outil " angulaire » $(\rho=0)$, de même valeur d'angle de coupe $\gamma$. La valeur $F_{n}=P_{o}$ pour $t / \rho=1$ apparaît notamment comme un seuil pour la charge verticale, en deçà duquel l'outil porte sur l'arête de coupe, et au delà duquel il porte sur la face en dépouille. L'expérience avec un outil $(\boldsymbol{\gamma}=0$, $e=1,5 \mathrm{~cm}, \rho=0,3 \mathrm{~cm}$ ) pour lequel $\mathrm{P}_{\mathrm{o}} \approx 20$ daN illustre bien cet " effet de pointe ». (fig. 10).

\section{Interprétation des résultats}

Les effets comparables sur le processus de destruction des roches par un outil de forme "angulaire», défini par le paramètre $\boldsymbol{\gamma}$, et un outil émoussé, défini par le paramètre $\rho / t$, suggèrent d'établir l'équivalence entre ces deux outils, sur la base de l'égalité des efforts de coupe (à l'influence du frottement de contact près) (fig. 11).

Un outil de forme "angulaire», avec angle de coupe $\gamma$ variant de $-\pi / 2 \mathrm{a}+\pi / 2$ est donc susceptible de représenter des outils de forme très variée. Un modèle théorique a été proposé pour un tel outil $[6,7]$; il donne des solutions cinématiques pour la structure supposée rigide, parfaitement plastique obéissant au principe du travail maximal, et composée du matériau de Coulomb non pesant, et de I'interface de contact OA rugueux (loi de frottement sec de Coulomb).

Pour l'outil avec arête de coupe arrondie, il est cependant possible d'obtenir des renseignements supplémentaires, en faisant un calcul approché, suggéré par l'observation du sens de l'écoulement du matériau en contact sur un tel outil et sur l'outil de forme " angulaire " (Fig. 12) :

en tout point du contact, on suppose que le sens de l'écoulement ainsi que les contraintes induites, $\sigma_{n}, T_{n t}$ sont les mêmes que ceux d'un outil " angulaire » dont la face d'attaque a la même inclinaison que le plan tangent en ce point. L'écoulement vers l'arrière aurait lieu pour $0 \leqslant \gamma^{\prime} \leqslant \pi / 4-\varphi / 2$

Les figures 13 et 14 montrent que les valeurs expérimentales sont plus proches de la courbe correspondant au frottement de contact maximal, qui correspond ici à l'agglomération du matériau au contact $([\vec{V}]=\overrightarrow{0})$. Dans la pratique, il peut arriver que le frottement de contact soit très faible (cas du polissage réciproque, effet de certains fluides de forage...): $c^{\prime}$ est le cas pour l'expérience de rayure du verre par un diamant (fig. 8). L'efficacité de la destruction pourrait alors être accrue si le frottement de contact pouvait être augmenté.

Les expressions des solutions obtenues sont données en Annexe 1: Les valeurs de $\mathscr{T}$ :et $\mathcal{N}$ obtenues tendent vers l'infini avec $\rho / \mathrm{t}$. La même remarque que précédemment (§ 2.2) conduit à borner ces valeurs par celles relatives au mode 2 (abrasion)

\section{Conclusion}

La face d'attaque et celle en dépouille d'un outil élémentaire ne jouent aucun rôle tant qu'un certain seuil $P_{0}$ n'a pas été franchi par la charge normale $F_{n}$ appliquée. Ce seuil augmente avec la résistance de la roche, l'usure de l'outil, la pression effective de confinement $\mathrm{P}_{\mathrm{e}}$ de la roche ; il reste cependant bien inférieur à la charge de poinçonnement statique de l'outil.

Ce dernier se comporte donc comme un outil " angulaire » d'angle de coupe $\gamma$ très faible (en valeur algébrique). L'écoulement vers l'arrière du matériau en contact est alors prépondérant : le frottement de contact n'a un effet diffé-


Fig. 9 Variation de $T$ et $N$ avec $t / \rho$

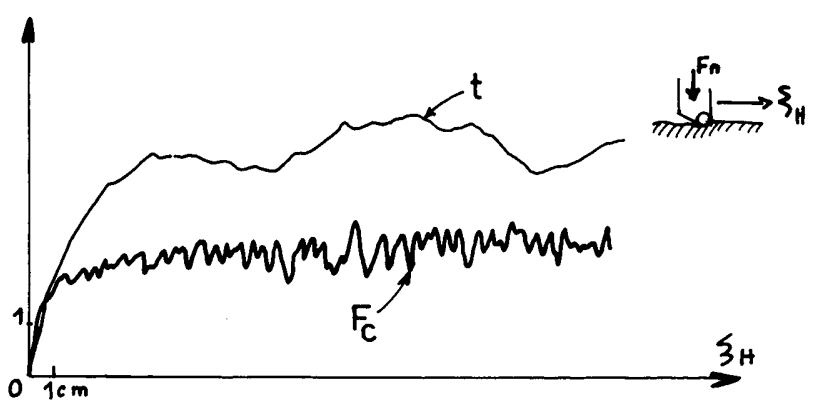

a) $F_{n}=15 \mathrm{daN} \quad\left(<P_{0}\right)$

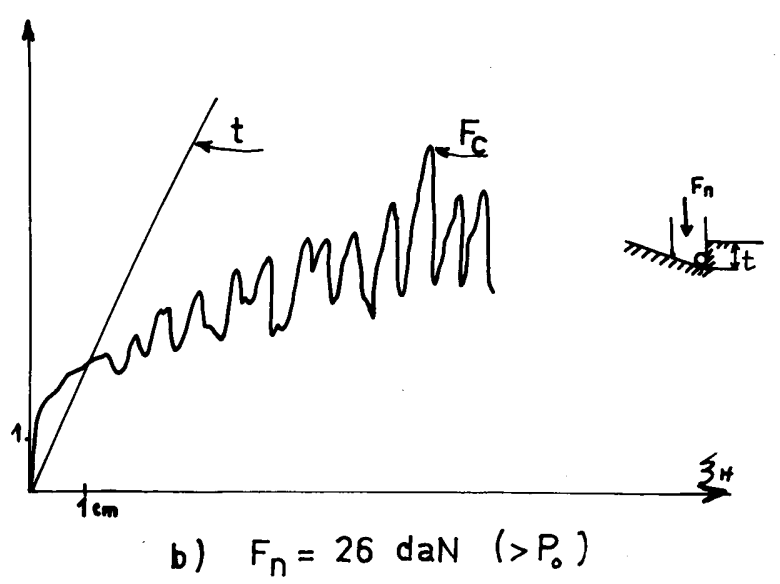

Fig. 10 Influence de Fn

$F c: 1 \mathrm{~cm}=5.5 \mathrm{daN}$

$t: 1 \mathrm{~cm}=0.5 \mathrm{~mm}$ 


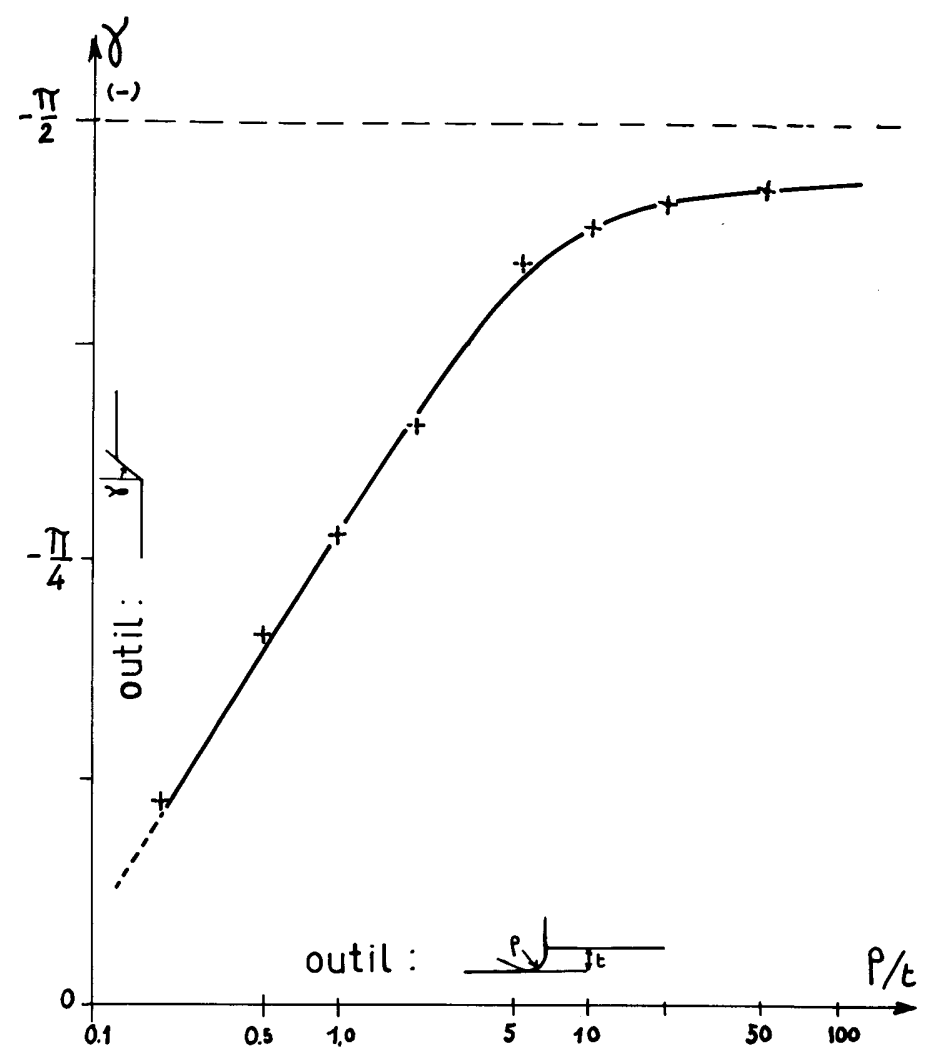

Fig. 11 Equivalence entre $\gamma$ et $\rho / t$



Fig. 12 Modèle théorique

rent sur la charge $F_{n}$ que dans le cas où l'écoulement se fait "vers le haut".

La connaissance des contraintes et du sens de l'écoulement relatif du matériau au contact sont des éléments importants pour l'étude du phénomène de l'usure. Le calcul approché, utilisé ici, peut être étendu au cas de l'outil de forme quelconque.

Enfin, la même méthode expérimentale peut être utilisée pour l'étude des cas où l'écoulement n'est pas plan leffet d'un bord libre, coupe oblique...), et pour lequel le calcul plastique n'est pas possible.

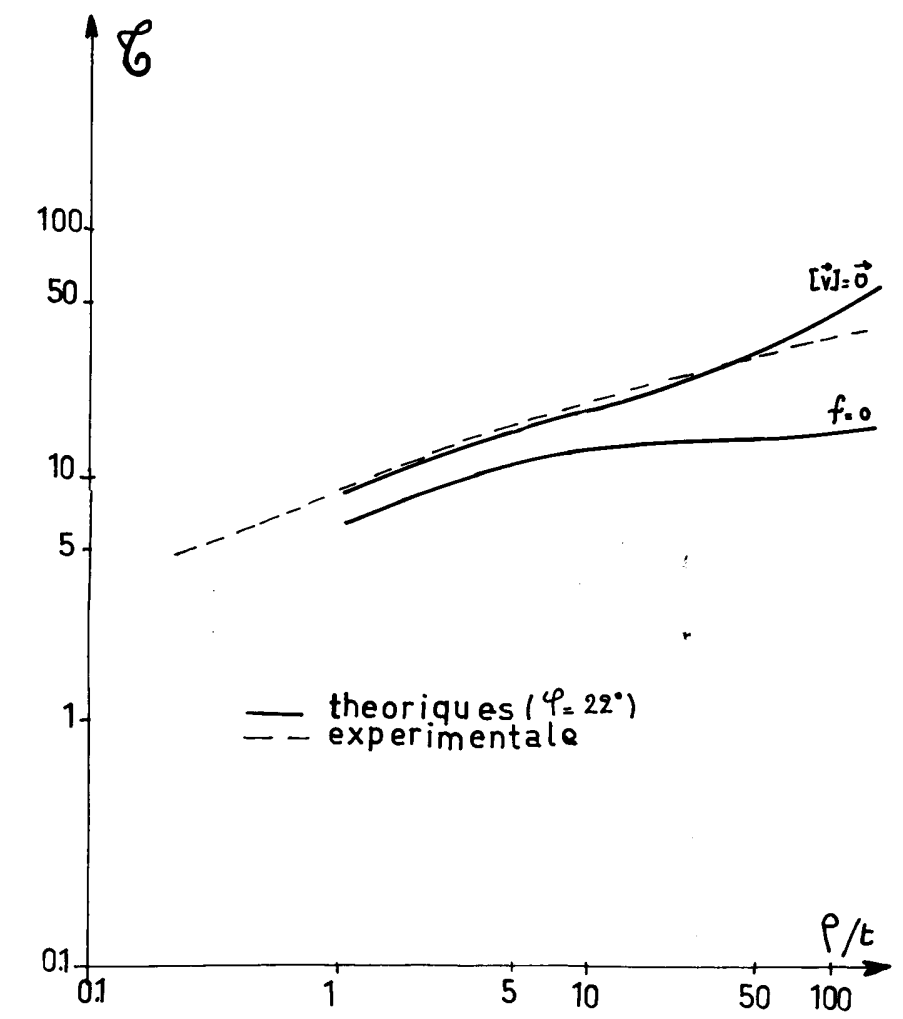

Fig. 13 Courbes composées ( $\mathscr{T}, \rho / t)$



Fig. 14 Courbes composées $(.1, \rho / t)$ 


\section{Références Bibliographiques}

[1] W. E. GARNER - Cutting action of a single diamond under simulated borehole conditions. Soc. Pet. Eng. I. p. 937/942. Juillet 1967.

[2] G. GAUTHIER, G. BARON. - Classement des roches préliminaires à une étude de forabilité. Étude bibliographique. Revue de l'Institut Français du Pétrole, Mars 1963.

[3] R. GOODRICH. - Rotary drilling Équipement. Bulletin of the University of Missouri School of Mines and Metallurgy, 1957, Technical series $n^{\circ} 94$, p. 25.

[4] K. E. GRAY. - Fixed blade planing of rocks in the brittle state. Ph D. Dissertation, Pet. Eng. Dept. Univ. of Texas Austin, 1963.

\section{Annexe}

On utilise l'approximation définie sur la figure 12 pour le calcul des contraintes et des efforts sur l'outil « circulaire». Les contraintes induites par l'outil "angulaire » sont données dans [6].

$\gamma^{\prime}=\hat{O C} M$ détermine la position du point courant $M$ sur la surface de contact, avec $\gamma^{\prime} \epsilon[0, \theta]$ et $\theta \varepsilon[0, \pi / 2]$.

on a :

$$
\frac{\rho}{t}=\frac{1}{1-\cos \theta}
$$

On donne ici les résultats pour deux cas extrêmes de contact.

\section{Frottement nul}

Les contraintes s'écrivent

$\left|\sigma_{n}\right|=H\left[\frac{(1+\sin \varphi)}{(1-\sin \varphi)} \cdot e^{(\pi-2 \gamma) \operatorname{tg} \varphi-1]}\right.$

$\tau_{\mathrm{nt}}=\mathrm{O}$

et les efforts réduits :
[5] P. HABIB, D. NGUYEN MINH. - Augmentation de la dureté des roches sous l'effet des contraintes tangentielles. Cahier $n^{\circ} 7$, Comité Français de Mécanique des Roches. Rev. Ind. Min., 1975.

[6] D. NGUYEN MINH. - Contribution à l'étude de la "taille " des roches. Thèse doct. ès Sciences, Université de Paris VI, 1974.

[7] D. NGUYEN MINH. - Contribution à l'étude de la "taille» des roches. Revue de l'Institut Français du Pétrole. Vol. XXX No 3 , Mai-Jùin 1975.

[8] Y. NISHIMATSU. - The mechanics of Rock Cutting. Int. J. Rock Mech. Min. Sc., 1972, Vol. 9, p. 261/270.

[9] L.A. SCHREINER, O.P. PETROVA. - Propriétés mécaniques et abrasives des roches. Moscou, 1958 trad. Neyfor (t. 1534).
Frottement maximal qui correspond ici à une agglomération de matériau au contact : $([\vec{v}]=\overrightarrow{0})$.

Les contraintes s'écrivent :

$$
\begin{aligned}
& \left|\sigma_{\mathrm{n}}\right|=(\rho+\mathrm{H})\left[1+\sin \varphi \sin \left(2 \gamma^{\prime}+\varphi\right)\right]-\mathrm{H} \\
& \tau_{\mathrm{nt}}=-(\rho+\mathrm{H}) \sin \varphi \cos \left(2 \gamma^{\prime}+\varphi\right) \\
& \text { avec } \quad \rho+\mathrm{H}=\frac{\mathrm{H}}{1-\sin \varphi} \mathrm{e}^{(\pi / 2+\varphi) \operatorname{tg} \varphi}
\end{aligned}
$$

et les efforts réduits :

$$
\begin{aligned}
& \pi=\frac{A_{0}}{1+4 \operatorname{tg}^{2} \varphi}\left[1-e^{-2 \theta \operatorname{tg} \varphi}(\cos \theta+2 \operatorname{tg} \varphi \sin \theta)\right]-\operatorname{cotg} \varphi \\
& T=\frac{(\rho+H)}{C} \sin \varphi \frac{\sin \varphi(\theta+\varphi)-\sin \varphi}{1-\cos \theta}+\frac{P}{C} \\
& R=\frac{A_{0}}{1+4 \operatorname{tg}^{2} \varphi}\left[2 \operatorname{tg} \varphi-e^{-2 \theta \operatorname{tg} \varphi}(2 \operatorname{tg} \varphi \cos \theta-\sin \theta)\right]-\frac{\operatorname{cotg} \varphi \sin \theta}{1-\cos \theta} \sin \varphi \frac{\cos \varphi-\cos (\theta+\varphi)}{1-\frac{P}{C}} \\
& \text { avec } \quad A_{0}=\frac{\operatorname{cotg} \varphi}{1-\cos \theta}\left(\frac{1+\sin \varphi}{1-\sin \varphi}\right) e^{\pi \operatorname{tg} \varphi}
\end{aligned}
$$$$
1=\frac{(\rho+H)}{C} \sin \varphi \frac{\cos \varphi-\cos (\theta+\varphi)}{1-\cos \theta}+\frac{P}{C} \frac{\sin \theta}{1-\cos \theta}
$$ 